\title{
Handedness and social anxiety: using Bryden's research as a catalyst to explore the influence of familial sinistrality and degree of handedness
}

Scott M. Hardie, Lynn Wright, Lisa Clark

This is an Accepted Manuscript of an article published by Taylor \& Francis in Laterality: Asymmetries of Body, Brain and Cognition on 12 January 2016, available online:

http://wwww.tandfonline.com/10.1080/1357650X.2015.1131712 


\section{Handedness and Social Anxiety: Using Bryden's research as a catalyst to explore the influence of familial sinistrality and degree of handedness}

\section{Scott M. Hardie, Lynn Wright and Lisa Clark}

Evolutionary and Biological Approaches to Behaviour Research Group, Division of Psychology, Abertay University, Bell Street, Dundee, DD1 1HG, UK

Keywords: Social Anxiety, Handedness, Familial Sinistrality, Degree of Handedness

\section{Abstract}

Phil Bryden's work has impacted on many areas of laterality, including degree and measurement of hand preference, as well as influences of familial sinistrality. For example, Bryden (1977) is a well-cited and influential paper that remains relevant to this day. Inspired by this we extended our analysis of the relationship between handedness and anxiety in a number of ways. We used familial handedness and strength of handedness to examine their potential influences on anxiety, and extended our research by exploring their relationship to social anxiety, using the Social Phobia Inventory (SPIN). Inconsistent left-handers (ILH) were found to be more socially anxious. In all categories of SPIN except avoidance, ILH were significantly more anxious than consistent right- and left-handers. There were familial sinistrality differences between ILH with a first-degree left-handed relative (FS+) compared to ILH with no first-degree left-handed relative (FS-) on all categories of anxiety scores. Within FS+ participants, ILH had significantly higher anxiety scores, compared with consistent handers across all categories. This suggests that ILH's social anxiety may be influenced by a close left-handed relative. Inspired by examining Bryden's work for this special issue, we will continue to add both strength of preference and familial handedness to our work.

Key Words: Bryden, Handedness, Consistency, Social Anxiety, Familial Sinistrality

Address correspondence to: Scott Hardie, Division of Psychology, Abertay University, Bell Street, Dundee, DD1 1HG.

Tel: (01382) 308587 Fax: (01382) 308749 E-mail: s.hardie@abertay.ac.uk 


\section{Introduction}

Phil Bryden has been a major influence on researchers across a diverse range of areas in the field of laterality, and it should come as no surprise that this includes the PhD Thesis of one of the current authors (Wright, 2005) which cites nine of his papers. Taking one particular study as an example, our research on handedness has certainly benefited from Bryden's work on the measurement of handedness (Bryden, 1977), and we are interested in investigating the extent to which familial influences might also have an effect. Influenced in part by this classic paper, we seek to examine the extent to which there are familial handedness effects in our exploration of the relationship between hand preference and anxiety (e.g. Wright \& Hardie, 2012). In addition, we also make reference to Bryden's influence on the emerging focus of degree of handedness (e.g. Prichard, Propper \& Christman, 2013) and how this may relate to behaviour and personality (Hardie \& Wright, 2014).

Bryden (1977) is an extremely influential paper related to the measurement of hand preference, which currently has 364 citations in Science Direct. An indication of enduring impact is shown by the fact that 60 of these citations are since January 2011, including some of our own recent work (e.g. Hardie \& Wright, 2014) and there are four citations so far in 2015. A very pertinent recent example is the work of Edlin et al. (2015) who have illustrated some major issues in terms of the way that the Edinburgh Handedness Inventory (Oldfield, 1971) is currently being used, including the choice of items and the scoring system - areas familiar to readers of Bryden's work (e.g. Steenhuis \& Bryden, 1991; McManus \& Bryden, 1992). For the present work, we note that Bryden (1977) endorsed the use of questionnaires to measure handedness and also examined the relationship between hand preference in an individual and the preferences of their close relatives.

Questionnaires are the most common method used to determine hand preference (McManus \& Bryden, 1992) and these have the advantage of allowing for the quantification of degree of handedness (Steenhuis \& Bryden, 1989; Bryden \& Steenhuis, 1987). During the last 10 years there has been a resurgence of interest in the concept of degree of handedness, culminating in a paper by Prichard et al. (2013), which argues that strength but not direction is the key. This serves as a useful summary of the importance of consistency of handedness as a variable of interest, but Hardie and Wright (2014) have subsequently demonstrated that at least in some cases, both strength and direction are important. Hardie and Wright go on to show that the relationship between consistency of handedness and behavioural inhibition can also show robust directional effects, where consistent left-handers show the highest levels of inhibition. Measuring both direction and strength is of course not a new concept, as some 25 years ago Steenhuis, Bryden, Swartz and Lawson (1990) advocated a view of handedness that is multidimensional, and included both aspects. There has been much debate 
regarding classifying consistency of handedness (see Hardie \& Wright, 2014 and also Edlin et al., 2015 for recent discussion), that is the extent to which individuals make used of their preferred hand. The debate focusses on how the EHI is used, and where the cut-off point should be in order to determine consistent and inconsistent classification.

An examination of familial influences on handedness has been ongoing for over 40 years and is thought to reflect hereditary factors (e.g. Annett, 1970; Reiss \& Reiss, 1999). Although the field has covered a wide range of definitions (Karev, 2011), the main focus has been on the presence of lefthanded (or non-right-handed) biological relatives. This has led to the concept of Familial Sinistrality (FS) where individuals are generally split into a dichotomous classification, with FS (however defined) being present (FS+) or absent (FS-) from that individuals' family (e.g. Bryden, 1977). A persistent problem throughout the literature is precisely which relatives to include in this split. For example, Nebes and Briggs (1974) confined their FS+ classification to those individuals who reported at least one non-right-handed sibling and/or parent. However, other researchers have extended this to include grandparents (e.g. Rebai et al., 1997), and even cousins (Salmaso \& Longoni, 1985).

What do we know about familial handedness? Bryden (1987) argued that there is a strong heritability for degree of handedness, but direction is less clear-cut. McManus and Bryden (1992) reviewed a number of aspects of how familial handedness may relate to individual handedness, and amongst this was the idea that maternal handedness was a stronger influence that paternal handedness. McKeever (2000) refined this by showing that maternal influences on left-handedness were more apparent in sons compared to daughters. In terms of relating FS to behaviour, results have been somewhat mixed.

Other studies have suggested that familial influences on handedness may be less important for lefthanders but may be significant for right handers. For example, Snyder and Harris (1993) looked at both consistency of handedness and familial influences on mental rotation and 3-dimensional drawing tests, and concluded that handedness effects were only found for males. Cerone and McKeever (1999) found that in right-handed females, FS- individuals have poorer performance in spatial tasks than FS+ individuals, but Ecuyer-Dab, Tremblay, Joanette and Passini (2005) found no influence of FS. It is certainly the case that gender and familial influences may interact differently and in the words of Lake and Bryden (1976: p269) "influences of the factors of FS (familial sinistrality) and sex appear to be significant but complex."

Recent work by Mellet et al. (2014) demonstrated that a non-maximal hand preference strength (i.e. did not score either \pm 100 on the EHI) in conjunction with a familial history of left-handedness, had a detrimental effect on verbal and spatial tasks. Sauerland and Gotzner (2013) compared right-handers 
who had no reported familial sinistrality (pure familial dextrals) with all other combinations, on a questionnaire asking participants to report numerical trivia based on their personal experience. They found that individuals with familial sinistrality avoided the use of exact numbers. Taken together, these recent studies suggest that FS is a variable that is worth exploring. Additionally, although there is continuing debate about the validity of using familial sinistrality as a measure (e.g. Bishop, 1990; Corey \& Foundas, 2005), in keeping with the focus of this special issue, we decided to investigate measures of social anxiety in the context of familial sinistrality, strength and direction of handedness in light of Bryden's work in this area (e.g. Bryden, 1977; McManus \& Bryden, 1992).

How may handedness relate to anxiety? Despite the strong evidence linking the right-hemisphere to negative affect (e.g. Davidson, 1992; Sutton \& Davidson, 1997), it is somewhat surprising that a clear, strong relationship between handedness and anxiety has not been established. Wright and Hardie (2012) examined methodological and other issues surrounding previous work (e.g. Beaton \& Moseley, 1984, 1991; Merckelbach, de-Ruiter \& Olff, 1989) and focused on the use of the State-Trait Anxiety Inventory (Spielberger, Gorsuch, Lushene, Vagg \& Jacobs, 1983). This makes the distinction between Trait Anxiety - a tendency to respond to situations in an anxious way, and State Anxiety - a transient emotional response towards the current situation, usually shown as subjective worry; apprehension and nervousness (see Wright \& Hardie, 2012). Although French and Richards (1990) had already used this measure, and found no differences, Wright and Hardie argued that there were methodological flaws, mainly surrounding the lack of a consistent event to stimulate or trigger state anxiety. Using the context of an experimental situation, Wright and Hardie (2012) found left-handers had significantly higher state anxiety scores, even when controlling for levels of trait anxiety. Building on this, Wright and Hardie (2015) found that state anxiety of left-handed females was higher than would be expected, when solving a novel puzzle. However, when the puzzle was no longer novel, female left-handers demonstrated the usual finding of state anxiety correlating with trait anxiety. For all males and male left-handers, there was a strong positive correlation between state and trait anxiety across both novel and repeated testing. This implies that for female left-handers, the act of completing a novel task in front of an experimenter is somewhat anxiety provoking, whether or not they are 'normally' an anxious person.

An additional focus in the relationship between handedness and anxiety has been to look not only at direction, but also degree of handedness. For example, nearly 40 years ago Hicks and Pellegrini (1978) found mixed- and left-handers were more anxious than right-handers. Wienrich, Wells and McManus (1982) added to this by showing that strongly right- and left-handed individuals expressed significantly more anxiety than those with a mixed or weak hand preference. However, Beaton and Moseley (1984; 
1991) failed to find any relationship between handedness and anxiety. Recently, Lyle, Chapman and Hatton (2013) found that consistent right-handers (CRH) had a higher level of anxiety than inconsistent right-handers (IRH) but handedness consistency did not influence the anxiety of lefthanders. Inconsistent left-handers (ILH) were found to show more anxiety than IRH individuals. This suggests that consistency of handedness is also worth examining in the context of anxiety.

Although there has been a resurgence in the examination of handedness and anxiety, the majority of studies (e.g. Wright \& Hardie, 2012; Lyle et al., 2013) have not specifically covered the concept of Social Anxiety, a fear of social situations and being around people in a social environment (Ruscio et al., 2008). Merckelbach et al. (1989) may offer a hint in this area - they report a near significant ( $p=$ .06) correlation between $\mathrm{EHI}$ handedness strength and the social phobia scale of the Fear Questionnaire (Marks \& Mathews, 1979). The female (81\%) dominated participant pool was strongly made up of right-handers (83\%), who reported the largest mean social phobia score, compared to lefthanders and mixed-handers. Testing for differences, they also suggest a marginally significant difference $(p=.06)$ when comparing 'pure' right-handers $(E H I+100)$ with a composite group made up of all others. This suggests that social phobia may differ between handedness groups, although in this case right-handers showed the highest levels, and this links to left-hemisphere involvement. This is somewhat puzzling, as most other work has linked the right-hemisphere (left-hand) to other aspects of anxious behaviour. For example, Choudhary and O'Carroll (2007) found left-handers displayed an increased prevalence of post-traumatic stress disorder (PTSD) symptoms, while Dillon (1989) showed that left-handers were more worried by factors such as how they perform in tests. Recently, Logue, Logue, Kaufmann and Belcher (2015) found that left-handers made up a disproportionately higher population of children presenting themselves for treatment in an urban mental health clinic in the USA. This demonstrated that left-handed children in this cohort had a greater severity of clinical symptoms including anxiety, and supports an association between left-handedness and anxiety. In relation to social awkwardness or shyness, recent unpublished work found that left-handers showed higher levels of shyness than right-handers (Kerr, 2015), while Spere Schmidt, Riniolo and Fox (2005) found that those high in shyness and sociability had a higher degree of mixed-handedness.

\section{Present Study}

Marks and Mathews' (1979) social phobia scale consists of questions linked to expressing discomfort in social situations for example, 'Being watched or stared at'; 'Speaking or acting to an audience' and 'Being criticized'. However, the present study focuses on the Social Phobia Inventory (SPIN - Connor et al., 2000) as it is a more up to date measure which strongly positively correlates with the social phobia scale and has its' own three subscales (Physiological Distress, Fear and Avoidance). The SPIN 
has been reported to have good reliability and validity (Antony, Coons, McCabe, Ashbaugh, \& Swinson, 2006). This allows a more fine grained investigation of the distinct aspects of anxiety provoked by social situations, and fits with the view that anxiety is a complex and multi-faceted concept (Corr, 2011). The current study aims to further examine the relationship between hand preference, gender and social anxiety with a focus on two areas of interest to M. P. Bryden - namely hand strength and familial influence.

\section{Method}

Participants

Four hundred and sixteen participants took part in this study, 102 were male and 314 were female. From this, two female participants with no overall direction of preference $(\mathrm{EHI}=0)$ were excluded prior to analysis. This left a subject pool of 414 participants with a mean age of 28.2 years, and a mean male age of 29.8 years (range 17-63) and female mean age of 27.7 years (range 17-71). Onehundred and eleven (26.8\%) were left-handed, 303 (73.2\%) were right-handed.

\section{Materials}

The questionnaire consisted of demographic factors including age, gender and an estimate of first degree familial handedness which made use of Questions 17,18, 23 and 24 from the Brief Handedness Questionnaire provided by McManus (http://www.ucl.ac.uk/medical-education/otherstudies/laterality/laterality-questionnaires/BriefHandednessQuestionnaire2009.pdf ). For these four familial handedness questionnaires, participants were given the following instruction: 'Please tell us about the handedness of your family. Only describe natural (that is, blood) relatives, not step-parents, or persons adopted or fostered. If you are not certain about someone's handedness, do not guess, but say 'Not sure'. Specific questions covered parents and siblings and took the format 'Is (or was) your mother right- or left-handed? '

A modified version of Oldfield's (1971) Edinburgh Handedness inventory (EHI) was also presented (Hardie \& Wright, 2012), which contained ten questions about hand preference. These consisted of the original ten handedness items as outlined in Oldfield's Appendix II (Writing, Drawing, Throwing, Scissors, Toothbrush, Knife (without fork), Broom (upper hand), Striking match (match), Opening box (lid), Spoon). Unlike the original scoring system of the EHI, we used a five point Likert scale for scoring responses (Hardie \& Wright, 2013), which is similar to the system used by other researchers (e.g. Christman, Propper \& Dion, 2004). Each question was answered on a five point Likert scale and 
responses were scored right always $(+10)$, right mostly $(+5)$, either $(0)$, left always $(-5)$, left mostly $(-$ 10). This format has been used in our previous work (e.g. Hardie \& Wright, 2013), and allows an examination of both strength and direction of handedness.

Participants were also asked to complete the Social Phobia Inventory (SPIN - Connor et al., 2000), a 17-item questionnaire with questions such as 'I am afraid of people in authority' which were answered on a five point Likert scale ranging from not at all (0) to 'extremely' (4) and yielded a score from 0 to 68, where a higher score indicates greater social anxiety. There are three SPIN subscales; a 6-item fear scale $(1,3,5,10,14,15)$, a 7 -item avoidance scale $(4,6,8,9,11,12,16)$ and a 4 -item physiological distress scale $(2,7,13,17)$.

\section{Procedure}

Through emails, website notices, sign-up sheets and face-to-face recruitment, participants were recruited from both a university and the general public, where they were asked to consent to take part. The recruitment initially started out with a general call for participants, and we did not specifically make reference to 'anxiety' in our recruitment, to try to limit self-selection of participants on the basis of real or perceived anxiety. For example, we used the following text "We are in the process of collecting data on the influence of handedness on aspects of personality and we are in need of more participants, to make sure that the results are as accurate as possible. Handedness is a useful predicator of behaviour, as it tells us something about the way that the two sides of the brain interact with each other. For a number of years we have been working on this relationship and are particularly interested in how the degree of hand preference can relate to how individuals interact with the world around them". During the course of recruitment, we made a specific attempt to recruit more lefthanders and for these purposes added titles (e.g. 'left-handers needed' or 'males required') and we added another sentence to recruitment text, such as "In particular we are in need of left-handers, but it would be wonderful to get data from anyone, so that we can cover all types of hand preference".

Once initially recruited, participants were sent a link to an on-line version of the questionnaires via email. This version first asked participants to answer the demographic and familial handedness questionnaires, followed by the EHI and SPIN in a randomised order. The research abided by the British Psychological Society Code of Human Research Ethics and was approved by our School Research Ethics Committee. 


\section{Data Processing}

If only one piece of data was missing from the $\mathrm{EHI}$, an average value was calculated for that participant (i.e. the other nine answers added then divided by 9, then rounded to the nearest value on the scale) and substituted for the missing data. If two or more data points were missing for a single participant, then their data was removed. In total, the data from four participants were removed due to missing data. As mentioned above, data from two participants who had no specific hand preference $(E H I=0)$ were excluded from analysis as direction (left or right) could not be assigned.

\section{Handedness Classification}

Scores on the EHI were added together, giving a possible range of -100 (completely left-handed) through to +100 (completely right-handed). We considered a score of less than 0 to indicate a lefthanded preference, and a score greater than 0 as indicative of a right-handed preference. In order to divide our sample according to consistency of handedness, and in a way comparable with other studies, we used the widely reported median EHI score of 80 (e.g. Christman \& Butler, 2011; Lyle \& Osborn, 2011) as our cut-off point. In terms of subsequent classification, we follow McManus (1996) in advocating a four categorical division (see Hardie \& Wright, 2014) ensuring that the sample is divided into consistent left-handers $(\mathrm{CLH})$, consistent right-handers $(\mathrm{CRH})$, inconsistent left-handers (ILH) and inconsistent right-handers (IRH). Both consistent groups included individuals who scored exactly on the cut-off point $( \pm 80)$.

\section{Categorisation of familial sinistrality}

Early work on familial sinistrality used a variety of methods and definitions (e.g. Kareve, 2011) and also included the use of the term ambidextrous (Bryden, 1977). As this term is used in an inconsistent way, and following McManus (2009), we decided to use a strict interpretation of familial sinistrality, only asking for left-handed, right-handed or 'not-sure' reports. The most commonly adopted method of Familial Sinistrality seems to be a definition of having at least one first degree relative (parents or siblings) who are left-handed (e.g. Orr, Cannon, Gilvarry, Jones, \& Murray, 1999). In the present study this is referred to as Familial Sinistrality (FS) and participants will be categorised as FS+ (reported presence of a first degree left-handed blood relative) or FS- (no reported first degree left-handed blood relative). Only specific reports of 'left-handed' are used to determine inclusion in FS+, otherwise participants are included in the FS- classification. 


\section{Results}

For each sub-scale of the SPIN and the Total SPIN score a 2 (gender) $\times 2$ (familial sinistrality) $\times 4$ (handedness category) between subjects ANOVA was conducted. The significant results and follow-up analyses of the significant effects are reported below. All SPIN scores are reported in Table 1.

\section{SPIN Total Anxiety Scores}

There was a significant effect of handedness category on Total SPIN scores $F(3,398)=4.55, p=.004$, partial $\eta^{2}=.03$, power $=.88$ and significant two-way interactions between gender and handedness category $F(3,398)=3.19, p=.02$, partial $\eta^{2}=.02$, power $=.74$ and familial sinistrality and handedness category $F(3,398)=5.47, p=.001$, partial $\eta^{2}=.04$, power $=.94$.

For the significant main effect of handedness category, Bonferroni post hoc analyses demonstrated that inconsistent left-handers were significantly more socially anxious than both consistent lefthanders $(p=.008)$ and consistent right-handers $(p=.014)$.

The significant gender $\mathrm{x}$ handedness category interaction was examined further. There was a significant difference between the SPIN Total scores in the handedness categories of males $F(3,98)=$ $3.29, p=.02$. Bonferroni post-hoc tests revealed that inconsistent left-handed males were significantly more socially anxious than consistent right-handed males $(p=.045)$.

The significant familial sinistrality $\mathrm{x}$ handedness category interaction was also further examined and there was a significant difference between the SPIN Total scores of the FS+ handedness groups ( $p$ $<.001)$. Bonferroni post hoc analyses found significant SPIN Total score differences between FS+ inconsistent left-handers and FS+ consistent right-handers $(p=.002)$, with inconsistent left-handers having significantly higher scores (30.6) than consistent right-handers (20), and between FS+ inconsistent left-handers and FS+ consistent left-handers $(p=.001)$, again inconsistent left-handers had significantly higher SPIN Total scores (30.6 and 15 respectively).

The differences between the familial sinistrality groups (FS+ vs. FS -) were also examined across each handedness category for SPIN Total scores. There was a significant difference between FS+ and FSinconsistent left-handers $(p=.002)$ with FS+ inconsistent left-handers scoring significantly higher than FS- inconsistent left-handers. 


\section{SPIN Physiological Distress}

For physiological distress scores there was a significant main effect of handedness category $F(3,398)=$ $7.88, p<.001$, partial $\eta^{2}=.06$, power $=.99$, and significant interactions between gender and handedness category $F(3,398)=6.04, p<.001$, partial $\eta^{2}=.04$, power $=.96$, and familial sinistrality and handedness category $F(3,398)=3.77, p=.01$, partial $\eta^{2}=.03$, power $=.81$.

The significant handedness category effect was further analysed using Bonferroni post hoc tests. Inconsistent left-handers showed more physiological distress than consistent right-handers $(p=.001)$, consistent left-handers $(p<.001)$ and inconsistent right-handers $(p=.04)$. The inconsistent lefthanders scored significantly higher in SPIN Physiological distress than each of the other groups.

Examining the gender $\mathrm{x}$ handedness interaction further there was a significant difference between the SPIN Physiological distress scores in the handedness categories of males $F(3,98)=7.44, p=.001$. Follow up analysis showed that for males there were significant differences between inconsistent lefthanders and the three other groups. Inconsistent left-handed males scores higher than consistent right-handers $(p=.001)$, consistent left-handers $(p=.001)$ and inconsistent right handers $(p=.001)$.

Gender differences were also examined within each handedness category. There was a significant SPIN Physiological distress score between male and female consistent left-handers ( $p=.022)$, with females scoring higher. However, when comparing male and female inconsistent left-handers, this time males were significantly higher $(p=.04)$.

The significant familial sinistrality $x$ handedness category interaction was also further examined. There was a significant difference between the SPIN Physiological distress scores of the FS+ handedness groups $(p<.001)$. FS+ inconsistent left-handers had significantly higher scores than consistent righthanders $(p=.02)$, consistent left-handers $(p<.001)$ and inconsistent right-handers $(p=.002)$.

The differences between the familial sinistrality groups (FS+ vs. FS -) were examined across each handedness category for SPIN Physiological distress scores. There was a significant difference between the FS+ and FS- inconsistent left-handers $(p=.04)$ with FS+ inconsistent left-handers scoring significantly higher and between the FS+ and FS- consistent left-handers $(p=.002)$ with the FS - group scoring higher. 


\section{SPIN Fear}

Again there was a significant main effect of handedness category $F(3,398)=3.92, p=.009$, partial $\eta^{2}=$ .01 , power $=.83$. There was a significant gender $\mathrm{x}$ handedness category interaction $F(3,398)=3.17, \mathrm{p}$ $=.02$, partial $\eta^{2}=.02$, power $=.73$ and a significant familial sinistrality $\mathrm{x}$ handedness category interaction $\mathrm{F}(3,398)=6.24, \mathrm{p}<.001$, partial $\eta^{2}=.05$, power $=.96$.

Bonferroni post-hoc tests were carried out on the significant handedness category effect. Inconsistent left-handers showed significantly more fear than both consistent right-handers $(p=.02)$. and consistent left-handers $(p=.02)$.

The significant gender $x$ handedness category interaction was examined further. There was a significant overall difference between the handedness groups in males $(p=.04)$ for the SPIN Fear scores. However follow up pairwise comparisons revealed no significant differences between the specific handedness groups in males (although the differences between ILH and CLH and ILH and CRH approached significance (.07 for both).

Further analysis of the familial sinistrality $\mathrm{x}$ handedness category interaction was conducted. There was a significant difference between the SPIN Fear scores of the FS+ handedness groups $(p<.001)$. Bonferroni comparisons found that in the FS+ group, inconsistent left-handers scored higher in SPIN fear than consistent right-handers $(p=.001)$, consistent left-handers $(p=.003)$ and inconsistent righthanders $(p=.02)$.

Again the differences between the familial sinistrality groups (FS+ vs. FS -) were examined within each handedness category for SPIN Fear scores. There was a significant difference between the FS+ and FSinconsistent left-handers $(p<.001)$ with FS+ inconsistent left-handers scoring significantly higher.

\section{SPIN Avoidance}

The only significant effect for SPIN Avoidance was the interaction between familial sinistrality and handedness category $F(3,398)=4.48, p=.004$, partial $\eta^{2}=.03$, power $=.9$.

The significant two-way interaction between familial sinistrality and handedness category was further investigated. There was a significant difference between the SPIN Avoidance scores of the FS+ handedness groups $(p=.005)$. Bonferroni pairwise comparisons found that there was a significantly 
higher SPIN Avoidance score for the inconsistent left-handers compared with consistent right-handers $(p=.01)$ and consistent left-handers $(p=.023)$.

Finally, the differences between the familial sinistrality groups (FS+ vs. FS-) were examined across each handedness category for SPIN Avoidance scores. There was a significant difference between the FS+ and FS- inconsistent left-handers $(p=.002)$ with FS+ inconsistent left-handers scoring significantly higher.

\section{Discussion}

The current study examined the relationship between hand preference, gender and social anxiety. More specifically a number of sub-scales of social anxiety were considered namely avoidance, fear and physiological distress and these were analysed in relation to levels of familial sinistrality, gender and handedness strength and direction (creating four groups - inconsistent left-handers (ILH); Inconsistent right-handers (IRH); consistent left-handers (CLH) and consistent right-handers (CRH)). There were no significant main effects of gender on the SPIN total score nor any of the sub-scales. However there were significant differences between the handedness groups on fear, physiological distress and total scores. Further analysis revealed these differences were particularly influenced by the difference between ILH and both of the consistent handedness groups. There were also significant handedness category $\mathrm{x}$ gender interactions for physiological distress and total scores. In physiological distress scores the interaction was driven by the difference between ILH males and all of the other male handedness groups. For the Total SPIN score, male inconsistent left-handers were more socially anxious than consistent right-handers.

As expected from previous research, which reported that left-handers are more avoidant when initiating a novel task (Wright, Hardie \& Rodway 2004) and also in their self-reported levels of behavioural inhibition (Wright, Hardie \& Wilson, 2009; Hardie \& Wright, 2014), we found a handedness related difference. However, in this case it was ILH that showed the highest levels of social anxiety. Consistent handers scored the lowest across all social anxiety measures, with this 
largely driven by consistent males. In addition, it does offer some partial support for the contention of Prichard et al. (2013), that consistency is more important than direction of handedness. On the other hand, the fact that the ILH were quite distinct in their high scoring supports Hardie and Wright's (2014) position that both strength and direction should be considered. It also fits with Willems, Van der Haegen, Fisher and Francks' (2014), plea for left-handers to be included and considered as an independent group. It is difficult to compare the current findings to other work regarding the influence of consistency of handedness on anxiety, as virtually all other work has only used two (right or left) or three handedness categories (Right, Left or Mixed). The only other study to date to make use of the four-category system was Lyle et al. (2013), who found that ILH were more anxious than IRH but there were no consistency differences within left-handers as a group. This was similar to the current findings where ILH had the highest levels of social anxiety, followed by IRH. Lyle et al. (2013) also found that $\mathrm{CRH}$ had higher levels of anxiety than IRH, which is in contrast to the current findings. It may be the case that different types of anxiety have independent causes (e.g. Weissman, 1988) and so it is important to conduct more research on the various types of anxiety using a similar consistency of handedness classification system (Hardie \& Wright, 2014).

How does consistency influence other types of behaviour? Prichard et al. (2013) reviewed a large body of literature on differences between consistent versus inconsistent handers. While the article strongly advocates that the distinction between direction is not very relevant (see Hardie \& Wright, 2014 and Beaton, Kaack \& Corr, 2015 for a critique of this approach), it does offer some insight into consistency of handedness findings that may be relevant for the current study. For example, inconsistent (mainly right-handers) have been shown to change their attitudes more than consistent handers, in response to a persuasive message (Christman, Henning, Geers, Propper, \& Niebauer, 2008), while inconsistent handers were also shown to be more risk aversive than consistent handers (Christman, Jasper, Sontam, \& Cooil, 2007). On the flip side there is a body of work which also suggests that consistent handers (mainly right) are more conservative in their outlook, demonstrating a higher degree of authoritarianism (Lyle \& Grillo, 2014; Christman, 2014), are more sensitive to disgust and are less 
sensation seeking (Christman, 2014), all of which might suggest that they are more rigid in their outlook and perhaps less likely to allow themselves to be influenced by others. Kimbrel, Cobb, Mitchell, Hundt, and Nelson-Gray (2008) also found that behavioural inhibition (measured as sensitivity to punishment) was positively associated with social anxiety. The present results would support the idea that left-handers who as a group show more behavioural inhibition (Wright et al., 2009) should also show more social anxiety, but is not consistent with the finding that consistent lefthanders are significantly higher in BIS than other handedness groups (Hardie \& Wright, 2014). Recently, Beaton et al. (2015) failed to find an influence of hand preference strength on levels of BIS, while confirming the relationship between left-handedness and higher levels of self-reported BIS. It may be that as consistent handers seem to be less worried about the effect of others, that being inconsistently handed (especially left-handed) may be what predisposes an individual to be most socially anxious. Taken together, these studies show that strength and direction of handedness may relate to anxiety in a complex way, which may not be too surprising when considering that anxiety is a complex multi-faceted construct (e.g. Corr, 2011). In addition, the present findings may have been influenced by the context of testing, as it was on-line and so had no particular event that might elicit anxiety. In previous work we have hypothesised that this may be a perquisite in order for left-handers to show differences in inhibitory behaviour (Wright \& Hardie, 2011; Wright \& Hardie, 2015). It may be the case that social anxiety differences may also only be found on state-like measures, requiring a context for social anxieties to be manifest in and this should be tested in the future.

Following Bryden (1977) we also brought in the variable of familial sinistrality (FS), and this was investigated along with handedness category on each sub-scale of the SPIN. There were no significant main effects of family sinistrality (FS) on any of the scores, but there were FS $x$ Handedness category interactions across all categories. In each case, the interactions were only significant for individuals who reported a first degree left-handed relative (FS+), and for all combinations inconsistent lefthanders (ILH) scored higher than both consistent left- and right-handers. In addition, for fear and physiological distress, ILH were also significantly more socially anxious than inconsistent right-handers. 
Further analysis revealed that within ILH, across all categories of social anxiety, individuals who have a first-degree left-handed relative (FS+) scored significantly higher compared to those who did not (FS-).

Previous work on FS has shown it to be an influence on several areas of behaviour, such as differences in cognitive processes (e.g. D'Andrea \& Spiers, 2005), including spatial skills (Cerone \& McKeever, 1999) and intelligence (Tan, 1998). Christman and Propper (2001) suggested that FS+ was also a marker for increased inter-hemispheric interaction, and it appears that work based on right-handers may support this. However, the influence of familial sinistrality on aspects of anxiety seems to be an under-researched area. To our knowledge there appears to be no work that specifically addresses this, so we present the current work as a preliminary investigation inspired by Bryden (1977). The main current finding is that familial handedness influences do seem to play a part in anxiety, especially for inconsistent left-handers. This should not be a surprise as research has shown that anxiety itself may be subject to familial influences. For example, Merikanagas et al. (1998) found that familial links in anxiety occurred, but were different according to the type of anxiety. A particularly interesting finding was that ILH with first-degree relatives show more anxiety compared to ILH with no first-degree lefthanded relatives. It would appear that left-handers with a weak hand-preference and a left-hander in their close family somehow show a higher degree of social anxiety than those without a similar relative. This points towards a possible social influence, and it is clear that handedness and related behaviours may be shaped by environmental factors (Schaafsma, Riedstra, Pfannkuche, Bouma \& Groothuis , 2009). If an individual is already a CLH then it is less likely that such an influence will have much of an effect. They are less likely to change their minds due to persuasion (e.g. Christman et al., 2008) and as they are already showing a strong consistent preference may be less swayed by the presence of another individual and /or their behaviour. On the other hand, for less strongly lateralised individuals the presence of another close relative with a left-handed preference and anxious 
behaviour may well be a more salient influence. This remains to be tested, but is an intriguing possibility.

In keeping with the spirit of this special issue, we feel that taking into account familial handedness as part of a questionnaire based approach (Bryden, 1977) has certainly been a worthwhile exercise. On the one hand, we are certainly aware that there is criticism of the whole notion of familial handedness having any real utility (e.g. Bishop, 1990), but equally we also aware of recent evidence which supports the idea that familial handedness may show some interesting findings when applied in conjunction with degree of preference (Mellet et al., 2014). By undertaking the current research we feel that we have achieved two main purposes. Firstly, we have reinforced our interest in the notion of familial influences on sinistrality and questioned whether having a first-degree left-handed relative influences social anxiety scores. At this point, we have some support for an influence, and some evidence that both direction and degree of preference may influence social anxiety. However, it is clear that we need to collect more data, especially from males, in order to ascertain whether these variables clearly influence anxiety in left-handers. This is important as previous work linking left-handers to negative affect has been mixed in terms of findings and this may be in part due to the heterogeneity of lefthanders in terms of their behaviour, background and preferences.

Secondly, it should be noted that although it is common to think about familial handedness as a marker for genetic influence (e.g. McManus \& Bryden, 1992; Bishop, 1990), an under researched aspect of familial sinistrality is the potential social influence of having left-handed family members interacting with an individual (Schaafsma et al., 2009). This may be especially pertinent when looking at the influence of parents on their children, and may also be gender dependent with left-handed mothers having a higher incidence of left-handed children compared with right-handed fathers (e.g. McManus, 1991). Karev (2011) argued that there may be 'psycho-sociological' influences contributing to sex differences in familial sinistrality and again we need to collect more data in order to better understand the behaviour of left-handers. 


\section{Limitations}

All scores were based on self-report via the internet, and this may have influenced results. Having said this, we do not think that any potential lack of accuracy or honesty in responding linked to the use of this format would be handedness category specific. Within each direction of handedness category (left or right), we have a similar proportion of consistent to inconsistent participants compared to other studies, although our overall proportion of left-handers is high (26.8\%). This is because as part of a mixed recruitment strategy we specifically advertised for left-handers, so the sample will have an element of self-selection. However, we did not directly mention anxiety when recruiting (we referred to it as a personality study) and so we do not consider that self-selection was based on perceptions or ideas realted to anxiety. Possibly of more concern, was that in common with many psychology researchers, we had a relatively low number of male participants. Males only represented around $25 \%$ of the total sample. Due to the analytical strategy creating 16 different sub-groups (Table 1 ) this means that the smallest group (FS+ CLH) had only 5 individuals and so the current differences based on consistency of handedness and familial sinistrality, particularly relating to gender, are preliminary and in need of further investigation. Finally, the validity of the SPIN may be called into question, and some researchers have suggested the use of a modified version (e.g. Carleton et al., 2010).

Conclusion

To conclude, this paper examined the influences of handedness strength and familial sinistrality on social anxiety scores. Familial sinistrality has been neglected in handedness research in recent times and this special issue has been a fitting opportunity to re-examine this variable. Interestingly, participants with a first degree left-handed relative showed a different pattern of social anxiety. More specifically, ILH with a first degree left-handed relative displayed higher social anxiety levels than those with no first degree left-handed relatives or with any of the other FS+ handedness groups. This suggests that social anxiety in ILH's may be influenced by close left-handed relatives. With these differences in mind, it is important to consider the inclusion of familial handedness in future work. 


\section{References}

Annett, M. (1970). A classification of hand preference by association analysis. British Journal of Psychology, 61, 303-321.

Antony, M.M., Coons, M.J., McCabe, R.E., Ashbaugh, A., \& Swinson, R.P. (2006). Psychometric properties of the social phobia inventory: Further evaluation. Behaviour Research and Therapy, 44, 1177-1185.

Beaton, A.A., Kaack, I.H., \& Corr, P.J. (2015). Handedness and behavioural inhibition system/behavioural activation system (BIS/BAS) scores: A replication and extension of Wright, Hardie and Wilson (2009). Laterality, 20 (5), 585-603.

Beaton, A. A., \& Moseley, L. G. (1984). Anxiety and the measurement of handedness. British Journal of Psychology, 75, 275-278.

Beaton, A. A., \& Moseley, L.G. (1991). Hand preference scores and completion of questionnaires: Another look. British Journal of Psychology, 82 (4), 521.

Bishop, D.V. (1990). On the futility of using familial sinistrality to subclassify handedness groups. Cortex, 26 (1), 153 - 155.

Bryden, M. P., \& Steenhuis, R. E. (1987). Handedness is a matter of degree. Behavioral and Brain Sciences, 10, 266-267.

Bryden, M.P. (1987). Handedness and cerebral organization: Data from clinical and normal populations. In D. Ottoson \& D. Schalling (Eds.), The dual brain: Unified functioning and specialization of the hemispheres (pp. 55-70). Houndmills: Macmillan Press.

Bryden, M.P. (1977). Measuring handedness with questionnaires. Neuropsychologia, 15, 617-624.

Carleton, R.N., Collimore, K.C., Asmundson, G.J.G, McCabe, R.E., Rowa, K., \& Antony, M.M. (2010). SPINning factors: Factor analytic evaluation of the Social Phobia Inventory in clinical and nonclinical undergraduate samples. Journal of Anxiety Disorders, 24, 94-101. 
Cerone, L. J., \& McKeever, W. F. (1999). Failure to support the right shift theory's hypothesis of a "heterozygote advantage" for cognitive abilities. British Journal of Psychology, 90, 109-123.

Choudhary, C.J. \& O'Carroll, R.E. (2007). Left hand preference is related to posttraumatic stress disorder. Journal of Traumatic Stress, 20(3), 365-369

Christman, S. (2014) Individual differences in personality as a function of degree of handedness: Consistent-handers are less sensation seeking, more authoritarian, and more sensitive to disgust, Laterality: Asymmetries of Body, Brain and Cognition, 19:3, 354-367

Christman, S.D., \& Butler, M. (2011).Mixed-handedness advantages in episodic Memory obtained under conditions of intentional learning extend to incidental learning. Brain and Cognition, 77, $17-22$.

Christman, S.D., Henning, B., Geers, A.L., Propper, R.E., and Niebauer, C.L. (2008). Mixed-handed persons are more easily persuaded and are more gullible:Inter hemispheric interaction and belief updating. Laterality, 13, 403-426.

Christman, S. D., Jasper, J. D., Sontam, V., and Cooil, B. (2007). Individual differences in risk perception versus risk taking: Handedness and interhemispheric interaction. Brain and Cognition, 63, 5158

Christman S.D. \& Propper R.E. (2001) Superior episodic memory is associated with interhemispheric processing. Neuropsychology, 15(4), 607-16.

Christman, S.D., Propper, R.E., \& Dion, A. (2004). Increased interhemispheric interaction is associated with decreased false memories in a verbal converging semantic associates paradigm. Brain and Cognition, 56, 313-319.

Connor, K.M., Davidson, J.R.T., Churchill, E., Sherwood, A., Foa, E.B., \& Weisler, R.H. (2000). Psychometric properties of the Social Phobia Inventory (SPIN): A new self-rating scale. British Journal of Psychiatry, 176, 379-386. 
Corey, D.M., \& Foundas, A.L. (2005). Measuring Familial Sinistrality: Problems with Dichotomous Classification. Laterality, 10, 321-335.

Corr, P.J. (2011). Anxiety: Splitting the phenomenological atom. Personality and Individual Differences, 50 (7), 889-897.

D'Andrea, E. A., \& Spiers, M. V. (2005). The effect of familial sinistrality and academic experience on cognition in right-handed women. Neuropsychology, 5, 657663.

Davidson, R. (1992). Anterior cerebral asymmetry and the nature of emotion. Brain and Cognition, 20, 125-151.

Dillon, K. M. (1989). Lateral preference and students' worries: A correlation. Psychological Reports, 65, 496-498.

Ecuyer-Dab, I., Tremblay, T., Joanette, Y., \& Passini, R. (2005). Real-life spatial skills, handedness, and family history of handedness. Brain and Cognition, 57, 219-221.

Edlin, J.M., Leppanen, M.L., Fain, R.J., Hackländer R.P., Hanaver-Torrez, S.D., \& Lyle, K.B. (2015). On the use (and misuse?) of the Edinburgh Handedness Inventory. Brain and Cognition, 94, $44-$ 51.

French, C.C., \& Richards, A. (1990). The relationship between handedness, anxiety and questionnaire response patterns, British Journal of Psychology, 81 (1), 57.

Hardie, S.M., \& Wright, L. (2013). The relationship between Revised Reinforcement Sensitivity Theory (rRST), handedness and indecision. Personality and Individual Differences 55, 312-316

Hardie, S.M., \& Wright, L. (2014). Differences between left- and right-handers in approach/avoidance motivation: influence of consistency of handedness measures. Frontiers in Psychology, 5:134. doi: 10.3389/fpsyg.2014.00134

Hicks, R. A., \& Pellegrini, R. J. (1978). Handedness and anxiety. Cortex, 14, 119-121. 
Karev, G.B. (2011). Quantitatively assessed familial sinistrality in right-, mixed-, and left-handers. Laterality, 16 (1), 93-106.

Kerr, A. (2015). Shyness: Investigating personal experiences, social perceptions and predictive factors using a mixed-methods approach. Unpublished Honours Thesis.

Kimbrel, N. A., Cobb, A. R., Mitchell, J. T., Hundt, N. E., \& Nelson-Gray, R. O. (2008). Sensitivity to punishment and low maternal care account for the link between bulimic and social anxiety symptomatology. Eating Behaviors, 9, 210-217.

Lake, D.A., \& Bryden, M.P. (1976). Handedness and sex differences in hemispheric asymmetry. Brain and Language, 3, 266-282.

Logue, D.D., Logue, R.T., Kaufmann W.E., \& Belcher, H.M.E. (2015) Psychiatric disorders and lefthandedness in children living in an urban environment, Laterality, 20 (2), 249-256. DOI: $10.1080 / 1357650 \times .2014 .961927$

Lyle, K. B and Grillo, M.C. (2014). Consistent-handed individuals are more authoritarian. Laterality: Asymmetries of Body, Brain and Cognition, 19 (2), 146 - 163. DOI:10.1080/1357650X.2013.783044

Lyle, K. B., Chapman, L. K., \& Hatton, J. M. (2013). Is handedness related to anxiety? New answers to an old question. Laterality, 18, 520-535.

Lyle, K.B., \& Osborn, A.E. (2011). Inconsistent handedness and saccade execution benefit face memory without affecting interhemispheric interaction. Memory, 19, 613-624.

Marks I. M., \& Mathews A. M. (1979). Brief standard self-rating for phobic patients. Behavioral Research Therapy, 17, 263-267.

McKeever, W. F. (2000). A new family handedness sample with findings consistent with X-linked transmission. British Journal of Psychology, 91, 21-39. 
McManus, I.C. (1996). "Handedness," in The Blackwell Dictionary of Neuropsychology, eds. J. G. Beaumont, P. M. Kenealy, and M. J. C. Rogers, (Oxford: Blackwell), 367-376.

McManus, I.C. (1991). The inheritance of left-handedness. Ciba Foundation Symposium, 162, 251-281.

McManus, I.C., \& Bryden, M.P. (1992). The genetics of handedness, cerebral dominance and lateralization. Handbook of Neuropsychology, 6, 115-144.

Mellet, E., Jobard, G., Zago, L., Crivello, F., Petit, L., Joliot, M., et al. (2014). Relationships between hand laterality and verbal and spatial skills in $\mathbf{4 3 6}$ healthy adults balanced for handedness. Laterality, 19, 383-404. doi: 10.1080/1357650x. 2013.796965

Merckelbach, H., de Ruiter, C., \& Olff, M. (1989). Handedness and anxiety in normal and clinical populations. Cortex, 25, 599-606.

Merinkangas, K.R., Stevens, D.E., Fenton, M., Stolar, S., O'Malley, S.W., Woods, S.W., \& Risch, N. (1998). Co-morbidity and familial aggregation of alcoholism and anxiety disorders Psychological Medicine, 28, 773-788.

Nebes, R. D., \& Briggs, G. G. (1974). Handedness and the retention of visual material. Cortex, 10, 209214.

Oldfield, R. C. (1971). The assessment and analysis of handedness: The Edinburgh Inventory. Neuropsychologia, 9, 97-113.

Orr, K.G., Cannon, M., Gilvarry, C.M., Jones, P.B., \& Murray, R. M. (1999). Schizophrenic patients and their first-degree relatives show an excess of mixed-handedness. Schizophrenia Research, 39(3), 167-76.

Prichard E., Propper, R. E., \& Christman, S. D. (2013). Degree of handedness, but not direction, is a systematic predictor of cognitive performance. Front. Psychol. 4:9. doi: 10.3389/fpsyg.2013.00009 
Rebaï, M., Lannou, J., Bernard, C., Bonnet, C., \& Rochetti, G. (1997). Hemispheric asymmetries of visual evoked potentials in relation to spatial frequency, handedness and familial left-handedness. International Journal of Psychophysiology, 25, 85-95.

Reiss, M., \& Reiss, G. (1999). Earedness and handedness: distribution in a German sample with some family data. Cortex, 35, 403-412.

Ruscio, A.M., Brown, T.A., Chiu,W., Sareen, T. J., Stein, M.B., \& Kessler, R.C. (2008). Social fears and social phobia in the USA: Results from the National Comorbidity Survey Replication. Psychological Medicine, 38, 15-28

Salmaso, D., \& Longoni, A. M. (1985). Problems in the assessment of hand preference. Cortex, 21, 533549.

Sauerland, U., \& Gotzner, N. (2013). Familial Sinistrals Avoid Exact Numbers. PLoS ONE, 8(3): e59103. doi:10.1371/journal.pone.0059103

Schaafsma,, S.M., Riedstra, B.J., Pfannkuche, K.A., Bouma, A., \& Groothuis, T.G.G. (2009). Epigenesis of behavioural lateralization in humans and other animals. Phil. Transcripts of the Royal Society $B, 364,915-927$

Snyder, P.J., \& Harris, L.J. (1993). Handedness, Sex, and Familial Sinistrality Effects on Spatial Tasks. Cortex, 29, 115-34.

Spere, K.A., Schmidt, L.A., Riniolo, T.C., \& Fox, N.A. (2005). Is a lack of cerebral hemisphere dominance a risk factor for social "conflictedness"? Mixed-handedness in shyness and sociability. Personality and Individual Differences, 39, 271-281

Spielberger, C. D., Gorsuch, R. L., Lushene, R. E., Vagg, P. R., \& Jacobs, G. A. (1983). Manual for the State-Trait Anxiety Inventory (Form Y). Palo Alto, CA: Consulting Psychologists Press.

Steenhuis, R.E., Bryden, M.P., Schwartz, M., \& Lawson S. (1990). Reliability of hand preference items and factors. Journal of Clinical and Experimental Neuropsychology, 12, 921-930. $10.1080 / 01688639008401031$ 
Steenhuis, R.E., \& Bryden,M.P.(1989).Different dimensions of hand preference that relate to skilled and unskilled activities. Cortex, 25, 289-304.

Sutton, S.K., \& Davidson, R.J. (1997). Prefrontal brain asymmetry: A biological substrate of the behavioral approach and inhibition systems. Psychological Science, 8, 204-210. DOI:10.1111/j.1467-9280.1997.tb00413

Tan, U. (1988). The relationship between nonverbal intelligence, familial sinistrality and Geschwind scores in right-handed female subjects. International Journal of Neuroscience, 43, 177182

Weissman, M. M. (1988). The epidemiology of anxiety disorders: rates, risks and familial patterns. Journal of Psychiatric Research, 22, 99-114.

Wienrich, A. M., Wells, P. A., \& McManus, C. (1982). Handedness, anxiety and sex differences. British Journal of Psychology, 73, 69-72.

Willems, R.M., Van der Haegen, L., Fisher, S. E., \& Francks, C. (2014). On the other hand: including left-handers in cognitive neuroscience and neurogenetics. Nature Reviews Neuroscience, 15, 193-201 .

Wright, L., \& Hardie, S.M. (2015). Left-handers look before they leap: Handedness influences reactivity to novel Tower of Hanoi Tasks. Frontiers in Psychology, 6:58.

Wright, L., \& Hardie, S. M. (2012). Are left-handers really more anxious? Laterality, 17 (5), 629 - 642. doi: 10.1080/1357650X.2011.615126

Wright, L., \& Hardie, S. M. (2011). Not ready to sort it yet: Left-handers show behavioural inhibition on a manual sorting task. Laterality, 16 (6), 753 - 767. doi: 10.1080/1357650X.2010.521752

Wright, L., Hardie, S. M., \& Wilson, K. (2009). Handedness and behavioural inhibition: Left-handed females show most inhibition as measured by BIS/BAS self-report. Personality and Individual Differences, 46, 20 - 24. 
Wright, L. (2005). Response style differences between left- and right-handed individuals. PhD Thesis. Abertay University, Dundee.

Wright, L., Hardie, S., \& Rodway, P. (2004). Pause before you respond: Handedness influences response style on the Tower of Hanoi Task. Laterality, 9( 2), 133 -147. 
Table 1: SPIN total and sub-scale scores by handedness, familial sinistrality and gender.

\begin{tabular}{|c|c|c|c|c|c|c|c|}
\hline Handedness & $\begin{array}{c}\text { Familial } \\
\text { Sinistrality }\end{array}$ & Gender & $\mathbf{N}$ & SPIN Total & $\begin{array}{c}\text { Physiological } \\
\text { Distress }\end{array}$ & Fear & Avoidance \\
\hline \multirow[t]{5}{*}{ Consistent left-handers } & FS- & Female & 26 & $22.2(12.7)$ & $4.9(3.4)$ & $8.2(4.9)$ & $9.2(5.5)$ \\
\hline & & Male & 8 & $20.3(14.5)$ & $3.6(2.8)$ & $7.0(5.6)$ & $9.6(6.8)$ \\
\hline & FS+ & Female & 9 & $18(10.6)$ & $2.7(2.7)$ & $7.0(4.5)$ & $8.3(4.5)$ \\
\hline & & Male & 5 & $9.6(12.9)$ & $1.4(2.6)$ & $3.4(4.6)$ & $4.8(6.1)$ \\
\hline & & Total & 48 & $19.8(12.9)$ & $3.9(3.3)$ & $7.3(4.9)$ & $8.7(5.6)$ \\
\hline \multirow[t]{5}{*}{ Inconsistent left-handers } & FS- & Female & 20 & $17.0(14.2)$ & $4.2(3.7)$ & $5.8(5.0)$ & $7.0(6.1)$ \\
\hline & & Male & 11 & $23.2(13.5)$ & $6.2(4.1)$ & $7.7(4.5)$ & $9.3(5.7)$ \\
\hline & $\mathrm{FS}+$ & Female & 25 & $28.0(13.0)$ & $5.9(3.0)$ & $10.3(5.0)$ & $11.8(5.7)$ \\
\hline & & Male & 7 & $40.0(12.4)$ & $10(3.4)$ & $14.9(4.2)$ & $15.1(5.6)$ \\
\hline & & Total & 63 & $25.0(14.9)$ & $5.9(3.8)$ & $8.9(5.5)$ & $10.2(6.3)$ \\
\hline \multirow[t]{5}{*}{ Consistent right-handers } & FS- & Female & 81 & $22.7(12.7)$ & $4.9(3.5)$ & $8.2(4.8)$ & $9.5(5.7)$ \\
\hline & & Male & 16 & $18.7(14.5)$ & $3.5(3.5)$ & $6.7(5.3)$ & $8.5(7.1)$ \\
\hline & $\mathrm{FS}+$ & Female & 43 & $20.8(13.1)$ & $4.7(3.5)$ & $7.3(5.1)$ & $8.8(5.7)$ \\
\hline & & Male & 11 & $17.0(11.5)$ & $3.6(3.7)$ & $5.9(3.9)$ & $7.6(5.4)$ \\
\hline & & Total & 151 & $21.3(13.0)$ & $4.6(3.5)$ & $7.6(4.9)$ & 9.1 (5.8) \\
\hline \multirow[t]{5}{*}{ Inconsistent right-handers } & FS- & Female & 64 & $23.2(13.4)$ & $5.3(3.7)$ & $8.3(4.9)$ & $9.6(5.8)$ \\
\hline & & Male & 34 & $18.7(14.2)$ & $3.8(3.3)$ & $6.8(5.6)$ & $8.0(6.1)$ \\
\hline & $\mathrm{FS}+$ & Female & 44 & $24.3(12.5)$ & $6.4(3.5)$ & $8.2(4.6)$ & $9.8(5.7)$ \\
\hline & & Male & 10 & $23.8(14.0)$ & $4.2(2.2)$ & $7.4(5.7)$ & $12.2(7.3)$ \\
\hline & & Total & 152 & $22.6(13.4)$ & 6.0 (3.4) & $7.9(5.0)$ & $9.5(6.0)$ \\
\hline
\end{tabular}

